\title{
Effect of Vitamin-D Supplement in Patients Diagnosed as Chronic Rhinosinusitis with Vitamin-D Deficiency
}

\author{
Yosef H. Saeed Abdelkarim', Agila Al-Barasi ${ }^{2}$, Essam S. Hussien ${ }^{3}$ \\ ${ }^{1}$ Otolaryngology Department, Medical College, Benghazi University, Senior Specialist in ENT Department, Benghazi Medical Center, \\ Benghazi, Libya \\ ${ }^{2}$ Otolaryngology Department, Medical College, Benghazi University, Consultant in ENT Department, Benghazi Medical Center, Benghazi, \\ libya \\ ${ }^{3}$ Quality Control Department, Benghazi Medical Center, Benghazi, Libya
}

Email address:

ym20032004@gmail.com (Y. H. S. Abdelkarim), agila1969@yahoo.com (A. Al-Barasi), essamalshariff@yahoo.com (E. S. Hussien)

\section{To cite this article:}

Yosef H. Saeed Abdelkarim, Agila Al-Barasi, Essam S. Hussien. Effect of Vitamin-D Supplement in Patients Diagnosed as Chronic Rhinosinusitis with Vitamin-D Deficiency. International Journal of Otorhinolaryngology. Vol. 2, No. 1, 2016, pp. 1-4. doi: 10.11648/j.ijo.20160201.11

Received: October 29, 2015; Accepted: January 11, 2016; Published: July 5, 2016

\begin{abstract}
Introduction: Rhinosinusitis is a heterogeneous disease characterized by local inflammation of the upper airways and sinuses. The pathogenesis of chronic rhino sinusitis remains poorly defined. It was recently proposed that Vitamin D deficiency may explain some part of this pattern. The aim of study is to evaluate the effect of vitamin D supplement in patients diagnosed as chronic rhino sinusitis with vitamin D deficiency. Materials and Methods: Prospective study was performed on 57 patients diagnosed as chronic Rhinosinusitis and vitamin D deficiency. Patients received vitamin D3 tablet (1000 IU) daily for one month. Before and after drug intake all patients were asked to fill in a questionnaire in which they rated their overall symptoms. Results: (75.4\%) of patients cured from smell disturbance, $(22.8 \%)$ of patients cured from headache, $(21.1 \%)$ cured from nasal obstruction and only $7 \%$ of patients cured from nasal discharge. (64\%) of patients improve (not cured) from nasal obstruction symptoms, (63\%) of patients not cure from nasal discharge. Conclusions: Vitamin D supplement shows significant improvement in symptoms of chronic Rhinosinusitis mainly smell disturbance, Vitamin D could be use as adjuvant treatment for Chronic Rhino sinusitis.
\end{abstract}

Keywords: Chronic Rhino Sinusitis, Vit D, Nasal Obstruction

\section{Introduction}

Rhinosinusitis is a heterogeneous disease characterized by local inflammation of the upper airways and sinuses. Rhinosinusitis lasting longer than 12 weeks is classified as chronic rhinosinusitis. Chronic rhino sinusitis is one of the most common chronic diseases in adults in the United States and affects up to $15 \%$ of the population [1-4]. Clinically patients complain mainly of nasal obstruction, nasal discharge, headache and olfactory loss. Various theories on the etiology and pathogenesis of chronic rhino sinusitis, such as allergy, bacterial and fungal infections, and structural abnormalities, have been proposed, however, the pathogenesis remains poorly defined [5]. It was recently proposed that Vitamin D deficiency may explain some part of this pattern [6].

Vitamin D is a secosteroid hormone which is synthesized in the skin or derived from nutritional sources, primarily metabolized in the liver to 25-hydroxyvitamin D3 and subsequently in the kidney into a hormonally-active form, $1 \alpha$, 25-dihydroxyvitamin D3 (calcitriol) [7]. The classic role for vitamin D is regulation of calcium and phosphor homeostasis, Vitamin D is also involved in essential cell regulatory processes such as proliferation, differentiation, apoptosis, angiogenesis and inhibition of pro-growth/pro-survival signaling pathways in a wide variety of cell types [8]. Serum vitamin D levels are dependent on vitamin D intake, maternal vitamin D stores and cutaneous synthesis. Most foods have vitamin D content that is not adequate for normal plasma levels, and vitamin D fortification of food is not routine in many countries, so cutaneous production is the most important source. Several factors have significant effects on serum vitamin D levels, including season, sunlight exposure, age and 
$\operatorname{diet}[9,10]$.

The aim of our study is to evaluate the effect of vitamin D supplement in patients diagnosed as chronic rhino sinusitis with vitamin D deficiency.

\section{Material and Methods}

Prospective study was performed on 57 patients diagnosed as chronic Rhinosinusitis who referred to ENT clinic, Benghazi medical center, Benghazi- Libya in the period from March 2014 to May 2015.

Written consent was obtained from each patients before enrollment into the research project. Inclusion criteria include age more than 18 years old, patients met the diagnostic criteria for chronic Rhino sinusitis(nasal obstruction, nasal discharge, smell disturbance and headache/facial pain), and serum vitamin D deficiency $(<20 \mathrm{ng} / \mathrm{ml})$. Exclusion criteria include previous history of nasal and sinuses surgery or trauma and history of systemic or intranasal medication for pervious one month.

After primary assessment the epidemiologic data were collected, including the age and sex. All patients were subjected to complete history and examination including nasal endoscopic examination and nose and paranasal CT scan to confirm the diagnosis of chronic Rhinosinusitis. The serum vitamin D (serum 25-hydroxyvitamin D) level was obtained to confirm the vitamin D deficiency. $(<20 \mathrm{ng} / \mathrm{ml})$, normal level (20-50 ng/ml). [11]

Patients received vitamin D3 (cholecalciferol) tablet (1000 IU) [11] daily for one month. Before and after drug intake, all patients were asked to fill in a questionnaire in which they rated their overall symptoms (as cured, improve or not cure) on Visual Analogue Scale (VAS). Symptoms include nasal obstruction, nasal discharge, smell disturbance and headache/facial pain.

\section{Results}

Table 1. Comparison between different age in study group.

\begin{tabular}{lll}
\hline AGE & N0 & \% \\
\hline$<29$ & 12 & 21.1 \\
$29-45$ & 37 & 64.9 \\
45 & 8 & 14 \\
\hline
\end{tabular}

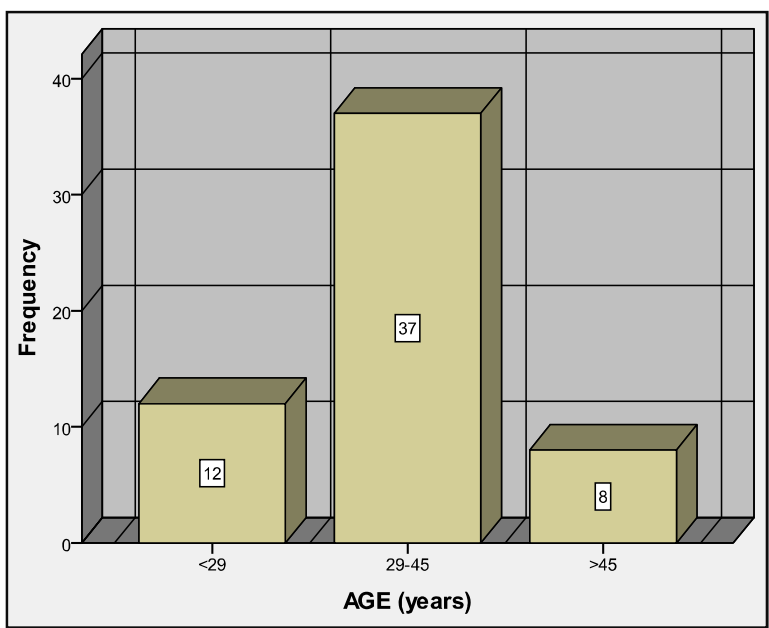

Figure 1. Comparison between different age in study group.

Table 2. Comparison between genders in study group.

\begin{tabular}{lll}
\hline gender & No & \% \\
\hline male & 4 & 7 \\
female & 57 & 93 \\
\hline
\end{tabular}

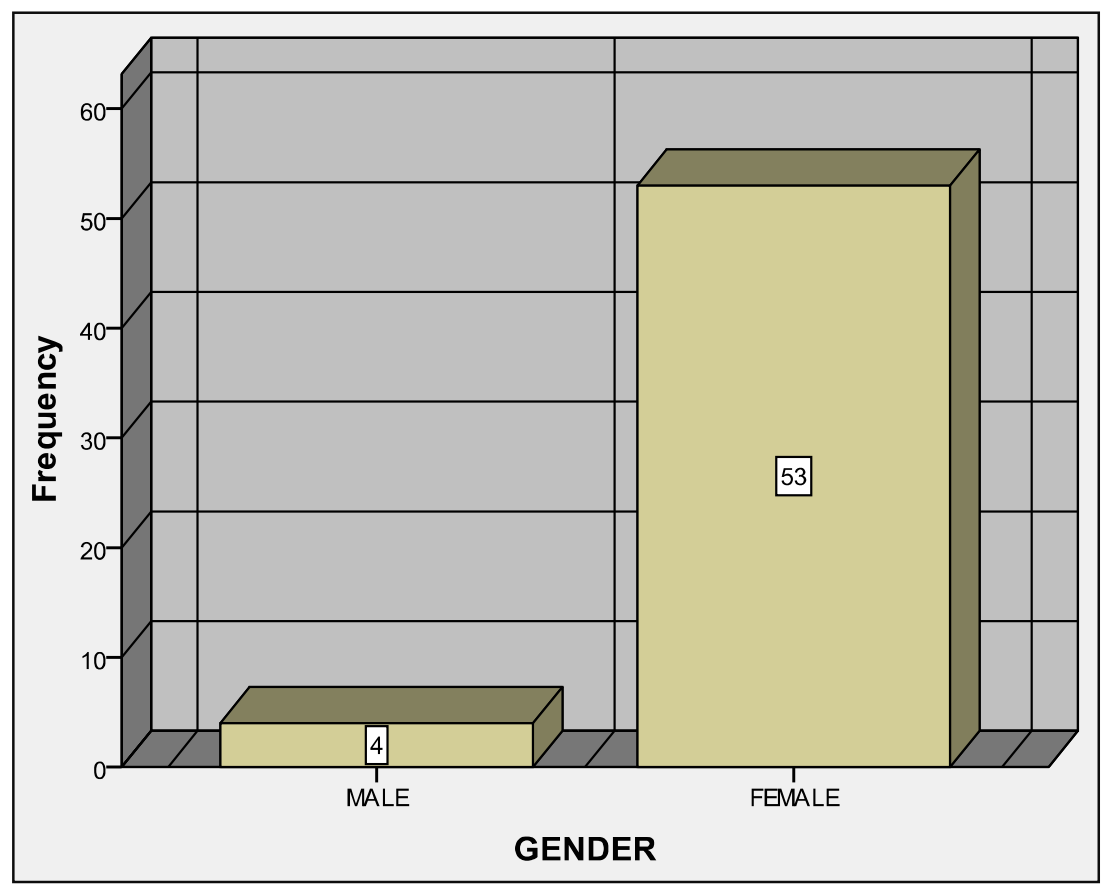

Figure 2. Comparison between gender in study group. 
Table 3. Comparison between different symptoms in study group.

\begin{tabular}{|c|c|c|c|c|c|c|c|c|}
\hline \multicolumn{2}{|c|}{ Headache/facial pain } & \multicolumn{2}{|c|}{ Smell disturbance } & \multicolumn{2}{|c|}{ Nasal discharge } & \multicolumn{2}{|c|}{ Nasal obstruction } & \\
\hline$\%$ & No & $\%$ & No & $\%$ & No & $\%$ & No & \\
\hline 22.8 & 13 & 75.4 & 43 & 7 & 4 & 21.1 & 12 & cure \\
\hline 59.6 & 34 & 17.5 & 10 & 29.8 & 17 & 64.9 & 37 & improve \\
\hline 17.5 & 10 & 7 & 4 & 63.2 & 36 & 14 & 8 & Not improve \\
\hline 114 & & 114 & & 114 & & 114 & & $\mathrm{X} 2$ \\
\hline 0.000 & & 0.000 & & 0.000 & & 0.000 & & P. value \\
\hline
\end{tabular}

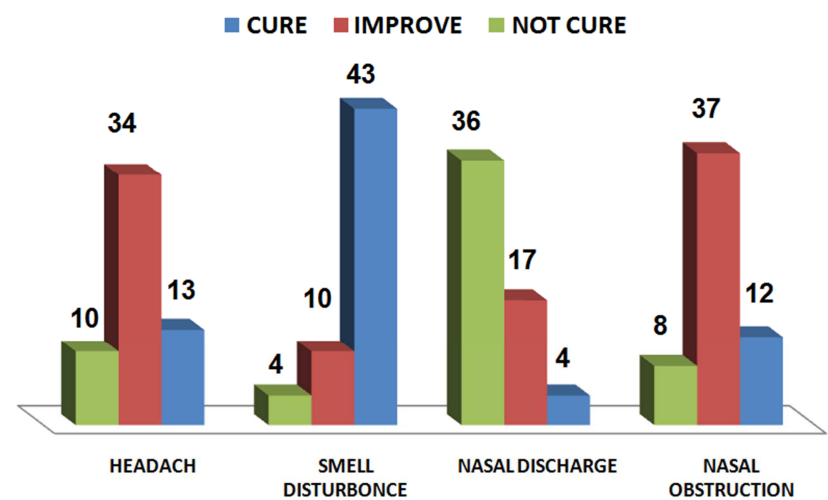

Figure 3. Comparison between different symptoms in study group.

\section{Discussion}

The underlying pathophysiology of chronic Rhinosinusitis is not necessarily infectious and is often a self-perpetuating inflammatory process. The predominant infiltrative cell in chronic Rhinosinusitis is the eosinophil, both in the allergic and the nonallergic patient, an increase in the levels of interleukin-4 and interleukin-5 in the sinonasal tract promotes the continued migration and prolonged life span of eosinophils, degranulation of eosinophils releases several destructive enzymes that damage the epithelium, this disrupts the normal barrier function and the mucociliary activity of the mucosa, allowing bacteria and fungi to colonize the sinus cavities [12]. Besides their classic role as regulators of calcium and phosphor homeostasis, vitamin D has emerged as a large family of antiproliferative agents; these observations suggest the potential of active vitamin $\mathrm{D}$ and its different derivatives and analogues as a therapy for chronic inflammatory diseases, including chronic Rhinosinusitis [13]. Several researches study the relation between chronic Rhinosinusitis and vitamin $\mathrm{D}$, Schlosser RJ et al [14]. study the impact of vitamin D in nasal polyposis and found that vitamin $\mathrm{D}$ insufficiency/deficiency is common in chronic sinusitis with polyposis patients, especially those of African American race. Wang LF et al [15]. also found that Serum 25-hydroxyvitamin D levels are lower in chronic rhinosinusitis with nasal polyposis. Abuzeid WM el al. [16] state that vitamin D may explain aspects of the pathophysiology of chronic Rhinosinusitis and may help direct future treatment of these diseases. Abdulbari B et al. [17] revealed that a high prevalence of Vitamin D deficiency in children with asthma and allergic diseases. Vitamin D deficiency was a strong correlate for asthma, allergic rhinitis and wheezing. Anna B et al. [18] found that low levels of $25(\mathrm{OH})$ Vitamin D might represent a risk factor for the development of concomitant asthma and rhinitis in children with allergic disease. J. K. Mulligan et al. [19] found that Vitamin D3 correlates inversely with systemic dendritic cell numbers and bone erosion in chronic rhinosinusitis with nasal polyps and allergic fungal Rhinosinusitis.

In our study we found that the age of patients diagnosed as chronic Rhinosinusitis with vitamin D deficiency were $(65 \%)$ range from $(29-45)$ years old, $(21 \%)(<29)$ years old and $(14 \%)(>45)$ years old. $(93 \%)$ were female patients while only (7\%) male, this may be because of clothing style (Hejab) and indoor living of Libyan women, that was also observed with the study done by Bahar C et al. [20] In current study we found that patients diagnosed as chronic Rhinosinusitis with vitamin $\mathrm{D}$ deficiency received one month course of vitamin D tablet, $(75.4 \%)$ of patients cured from smell disturbance, (22.8\%) of patients cured from headache, $(21.1 \%)$ cured from nasal obstruction and only $7 \%$ of patients cured from nasal discharge. (64\%) of patients improve (not cured) from nasal obstruction symptoms, (63\%) of patients not cure from nasal discharge. Statistical analysis showed very high significant difference $(P<0.001)$. Similar researches done. Beata $\mathrm{R}$ et al. [13] Study the Influence of vitamin D3 analogues in combination with budesonid treatment on proliferation of nasal polyp fibroblasts they state that the antiproliferative activity of calcitriol and tacalcitol in nasal polyposis cultures was confirmed. Because of its lower toxicity and higher activity tacalcitol seems to be the more promising agent in nasal polyposis therapy. Gong W et al. [21] Study the effect of nasal instillation of vitamin $\mathrm{D}$ on patients with allergic rhinitis and found that improve the symptoms for patients with allergic rhinitis. Vitamin D3 may be a kind of adjuvant therapy for prevention and treatment of allergic rhinitis. On contrary to Yawn J et al. [22] who reported that conflicting results of vitamin D supplementation trials, and potential gaps in our knowledge that may be limiting the widespread use of vitamin D for the treatment of respiratory diseases such asthma, chronic rhinosinusitis and allergic rhinitis.

\section{Conclusions and Recommendations}

- Chronic Rhinosinusitis with vitamin D deficiency are common among female than male.

- Vitamin D supplement shows significant improvement in symptoms of chronic Rhinosinusitis mainly smell disturbance.

- Vitamin D shows little improvement for nasal discharge. 
- Vitamin D could be use as adjuvant treatment for Chronic Rhinosinusitis.

- Further studies with more patients and longer duration of treatment are required for more evaluation.

\section{References}

[1] Meltzer EO, Hamilos DL, Hadley JA, Lanza DC, Marple BF, Nicklas RA, et al. Rhinosinusitis: establishing definitions for clinical research and patient care. Otolaryngol Head Neck Surg 2004; 131: S1-62.

[2] Kern RC, Conley DB, Walsh W, Chandra R, Kato A, Tripathi-Peters A, et al. Perspectives on the etiology of chronic rhinosinusitis: an immune barrier hypothesis. Am J Rhinol 2008; 22: 549-59.

[3] Schleimer RP, Kato A, Peters A, Conley D, Kim J, Liu MC, et al. Epithelium, inflammation, and immunity in the upper airways of humans: studies in chronic rhinosinusitis. Proc Am Thorac Soc 2009; 6: 288-94.

[4] Van Bruaene N, Derycke L, Perez-Novo CA, Gevaert P, Holtappels G, De Ruyck N, et al. TGF-beta signaling and collagen deposition in chronic rhinosinusitis. J Allergy Clin Immunol 2009; 124: 253-9.

[5] Dong Y, Seong H, Cho Tetsuji T, Robert P. Chronic Rhinosinusitis and the Coagulation System. Allergy Asthma Immunol Res. 2015 September; 7 (5): 421-430.

[6] Litonjua AA, Weiss ST. Is Vitamin D deficiency to blame for the asthma epidemic? J Allergy Clin Immunol 2007; 120: 1031-5.

[7] Holick MF (1984) The photobiology of vitamin D3 in man. Basic and Clinical Aspects. Kuman R, ed, pp 197-216.

[8] Johnson CS, Hershberger PA, Trump DL. Vitamin D-related therapies in prostate cancer. Cancer Metastasis Rev. 2002; 21: $147-158$.

[9] Hatun S, Islam O, Cizmecioglu F, et al. Subclinical vitamin D deficiency is increased in adolescent girls who wear concealing clothing. J Nutr 2005; 135: 218-222.

[10] Saadi HF, Dawodu A, Afandi B, et al. Effect of combined maternal and infant vitamin $D$ supplementation on vitamin $D$ status of exclusively breastfed infants. Matern Child Nutr 2009; 5: 25-32.

[11] Ross AC, Manson JE, Abrams SA, et al. "The 2011 report on dietary reference intakes for calcium and vitamin $\mathrm{D}$ from the Institute of Medicine: what clinicians need to know". J. Clin. Endocrinol. Metab. 96 (1): 53-8.
[12] Andrew P, Davied W: Sinusitis and polyposis. In James B, John JB. (Ed): Ballenger's Otorhinolaryngology, Head and neck surgery, 16th ed. ch. 26, spain, BC Decker Inc, 2003, pp276-77.

[13] Beata R, Marcin F, Wojciech G, Małgorzata L. Influence of vitamin D3 analogues in combination with budesonid $\mathrm{R}$ on proliferation of nasal polyp fibroblasts. Acta Biochimica Polonica. Feb2009; 56: 235-42.

[14] Schlosser RJ1, Soler ZM, Schmedes GW, Storck K, Mulligan JK. Impact of vitamin D deficiency upon clinical presentation in nasal polyposis. Int Forum Allergy Rhinol. 2014 Mar; 4 (3): 196-9. doi: 10.1002/alr.21274. Epub 2014 Jan 10.

[15] Wang LF1, Lee CH, Chien CY, Chen JY, Chiang FY, Tai CF. Serum 25-hydroxyvitamin D levels are lower in chronic rhinosinusitis with nasal polyposis and are correlated with disease severity in Taiwanese patients. Am J Rhinol Allergy. 2013 Nov-Dec; 27 (6): e162-5.

[16] Abuzeid WM1, Akbar NA, Zacharek MA. Vitamin D and chronic rhinitis. Curr Opin Allergy Clin Immunol. 2012 Feb; 12 (1): 13-7.

[17] Abdulbari B, Mohammad S., Hale Z, Qutayba H. The impact of Vitamin D deficiency on asthma, allergic rhinitis and wheezing in children: An emerging public health problem. Journal of Family and Community Medicine. 2014 Dec; 21 (3): 154-61.

[18] Anna B, Sebastiano G, Stefania L, et al. 25-Hydroxyvitamin D, IL-31, and IL-33 in Children with Allergic Disease of the Airways. Hindawi Publishing Corporation. June2014; 10: $1-10$.

[19] Mulligan J, Bleier B, Connell B, et al. Vitamin D3 correlates inversely with systemic dendritic cell numbers and bone erosion in chronic rhinosinusitis with nasal polyps and allergic fungal Rhino sinusitis. Clinical and Experimental Immunology. Jan 2011; 164: 312-320.

[20] Bahar Çuhacı-Çakır, Fatma Demirel. Effects of seasonal variation and maternal clothing style on vitamin D levels of mothers and their infants. The Turkish Journal of Pediatrics 2014; 56: 475-481.

[21] Gong W, Feng Y, Yan P, et al. Effect of nasal instillation of vitamin D3 on patient with allergic rhinitis symptoms. Lin Chung Er Bi Yan Hou Tou Jing Wai Ke Za Zhi. 2014 Jul; 28 (14): 1031-3.

[22] Yawn J, Lawrence LA, Carroll WW, Mulligan JK. Vitamin D for the treatment of respiratory diseases: is it the end or just the beginning? J Steroid Biochem Mol Biol. 2015 Apr; 148: 326-37. 